\title{
Microgloma Sanders \& Allen, 1973 (Nuculanidae) and Pristigloma Dall, 1900 (Pristiglomidae) (Pelecypoda) in the Campos Basin of Brazil
}

\author{
Natalia Pereira Benaim ${ }^{\dagger}$, Ricardo Silva Absalão ${ }^{\ddagger}$ \\ Universidade Federal do Rio de Janeiro, Departamento de Zoologia, Rua Professor Rodolpho Paulo Rocco, Ilha \\ do Fundão, CEP 21941901 \\ † urn:lsid:zoobank.org:author:CA4A4E78-CF51-47BD-AF67-3F6C1CFC6F52 \\ † urn:lsid:zoobank.org:author:D89BFB87-8BAF-44C4-AABD-5A40E202EE4C \\ Corresponding author: Natalia P. Benaim (nataliabenaim@gmail.com)
}

Academic editor: N. Yonow | Received 1 June 2011 | Accepted 20 September 2011 | Published 8 December 2011

urn:lsid:zoobank.org:pub:5B7582D0-AF05-4E9A-B7AC-F0428797E026

Citation: Benaim NP, Absalão RS (2011) New records of the genera Microgloma Sanders and Allen 1973 (Nuculanidae) and Pristigloma Dall 1900 (Pristiglomidae) (Pelecypoda) in the Campos Basin of Brazil. ZooKeys 152: 1-20. doi: $10.3897 /$ zookeys.152.1646

\begin{abstract}
As a secondary result of oil prospecting in Brazil, samples from the Campos Basin continental slope became available. In these samples, specimens of the genera Microgloma Sanders \& Allen, 1973 and Pristigloma Dall, 1900 were found. This contribution provides the southernmost record of the genus Microgloma, the first record of M. mirmidina (Dautzenberg \& Fischer 1897) from the western Atlantic, the descriptions of M. macaron sp. n. and $M$. nhanduti sp. n. as new species, and the shallowest record of Pristigloma alba Sanders \& Allen 1973.
\end{abstract}

\section{Keywords}

Pristiglomidae, Nuculanidae, Pelecypoda, deep-sea, biodiversity, Campos Basin slope, Rio de Janeiro

\section{Introduction}

Knowledge of the deep-sea mollusks from the Campos Basin has increased in the last ten years, and several new species have been described (Absalão et al. 2001, 2003; Absalão and Pimenta 2003, 2005; Caetano et al. 2006; Zelaya et al. 2006; Pimenta et al.

Copyright N.P. Benaim, R.S. Absalão. This is an open access article distributed under the terms of the Creative Commons Attribution License, which permits unrestricted use, distribution, and reproduction in any medium, provided the original author and source are credited. 
2008; Oliveira and Absaláo 2007, 2008, 2009, 2010a, 2010b; Absalão 2009; Benaim and Absaláo 2011). However, the genera Microgloma Sanders \& Allen, 1973 and Pristigloma Dall, 1900 have not been recorded until the present report.

Microgloma and Pristigloma comprise a few species that appear represented only in the Atlantic Ocean, each genus being represented by no more than five species. Their systematic affinities have been the subject of debate for almost 30 years (Sanders and Allen 1973, Allen and Hannah 1986, Ockelmann and Warén 1998, La Perna 2003, 2008), reflecting the usual confusion in protobranch taxonomy. Both Pristigloma and Microgloma were considered as members of the Pristiglomidae by Sanders and Allen (1973) and subsequent authors, until Ockelmann and Warén (1998) revised the systematic affinities of the genus Microgloma. This genus is characterized by miniaturization, and was considered by Sanders and Allen (1973) to be among the smallest pelecypods known. Synapomorphies include the enlarged innermost teeth of the left valve and the radially wrinkled surface of the prodissoconch. Members of this genus may comprise progenetic representatives of the family Nuculanidae, and may represent a polyphyletic group (Ockelmann and Warén 1998, La Perna 2008).

The five species of Microgloma described until now [M. tumidula (Monterossato, 1880), Microgloma mirmidina (Dautzenberg \& Fischer, 1897), Microgloma yongei Sanders \& Allen, 1973, M. pusilla Jeffreys, 1979, and M. guilornadi Hoeksema, 1993] were recorded from Western Europe (Iberian Peninsula, Mediterranean Sea, Azores and Canaries), West Africa (Cape Verde and Angola), Surinam, and North America. Prior to the report of Ockelmann and Warén (1998), there was no record of the genus Microgloma for the western Atlantic; these authors suggested the possibility of the presence of some specimens of Microgloma in Surinam, but made no formal record. Allen (2008) presented a checklist of the pelecypods of the Atlantic and made reference to the presence of $M$. pusilla, M. tumidula (as M. turnerae), M. yongei, and Microgloma sp. in North America and Surinam.

Pristigloma is a genus widespread in the entire Atlantic Ocean and is characterized by smooth, fragile shells, lamellar hinge teeth with an unequal number of teeth on both plates, and a large, internally elongated ligament which is opisthodetic (Sanders and Allen 1973).

Here, we present the first records of the genus Microgloma from the southwestern Atlantic, as well as descriptions of two new species belonging to this genus. We also present new points in the geographical distribution of Pristigloma alba Sanders \& Allen 1973.

\section{Material and methods}

The samples used in the present study were collected by means of a box corer in the Campos Basin, off Rio de Janeiro State $\left(22^{\circ} \mathrm{S}, 41^{\circ} \mathrm{W}\right)$, Brazil, from the research vessel Astro-Garoupa, as part of the programs "Environmental Characterization of Campos Basin, RJ, Brazil" during the years 2002 and 2003, and "Habitats Project - Campos 
Basin Environmental Heterogeneity" in 2008 and 2009. Both programs were sponsored by the Brazilian oil company Petrobras S.A. Of the material obtained we observed 260 samples taken between the isobaths of 400 and $2500 \mathrm{~m}$. The list of localities with Microgloma and/or Pristigloma specimens is given in Tables 1 and 2. Most of the shells were found in a good state of preservation, with valves attached, ligaments intact, and often with the mass of the animal body inside the shell. Unfortunately, there were no preserved organs in these cases. Each specimen was examined under stereoscopic microscope (Nikon SMZ 800), and selected specimens were photographed with a scanning electron microscope (ZEISS EVO 40), at the Gerência de Bioestratigrafia e Paleoecologia Aplicada (BPA), of the Petrobras Research Center (Centro de Pesquisas da Petrobras, CENPES).

Taxonomic identifications were made through comparison with the figures of type specimens [Microgloma pusilla (Jeffreys, 1879)] and descriptions available in the literature (Sanders and Allen 1973, Salas 1996, Ockelmann and Warén 1998, La Perna 2008, Oliver et al. 2009). The species were characterized considering traditional criteria used in pelecypod orientation and terminology (Figs 1-2) (Fischer 1886, Sanders and Allen 1973, Mikkelsen and Bieler 2008, Baylei 2009). In view of the importance of the features of the hinge plate for the discrimination of other protobranch species (Benaim and Absalâo 2011, Benaim et al. 2011), and also some subjective concepts in taxonomy (e.g., 'thin' or 'thick'), we described the species using certain quantitative criteria such as the ratios of the hinge teeth (wht) and hinge plate (whp) measurements (Figs 1-2), which are described as follows: 'thin' for width of hinge plate/total height ratio $<0.1$; 'thick' for width of hinge plate/total height ratio $\geq 0.1$. The width of the hinge teeth was measured just above (dorsal) and below (ventral) the limit of the big-

Table I. Table of the localities sampled as part of the project Environmental Characterization of Campos Basin.

\begin{tabular}{c|c|c|c|c}
\hline Station & Depth $(\mathbf{m})$ & Latitude & Longitude & Date \\
\hline 10 & 1700 & $21^{\circ} 58^{\prime} 36.06^{\prime \prime} \mathrm{S}$ & $39^{\circ} 46^{\prime} 30.28^{\prime \prime} \mathrm{W}$ & $08 / 10 / 2001$ \\
\hline 28 & 1930 & $22^{\circ} 06^{\prime} 52.98^{\prime \prime} \mathrm{S}$ & $39^{\circ} 44^{\prime} 13.90^{\prime \prime} \mathrm{W}$ & $08 / 05 / 2002$ \\
\hline 32 & 900 & $22^{\circ} 38^{\prime} 01.14 " \mathrm{~S}$ & $40^{\circ} 17^{\prime} 26.55^{\prime \prime} \mathrm{W}$ & $18 / 05 / 2002$ \\
\hline 33 & 900 & $22^{\circ} 35^{\prime} 47.22^{\prime \prime} \mathrm{S}$ & $40^{\circ} 15^{\prime} 00.33^{\prime \prime} \mathrm{W}$ & $18 / 05 / 2002$ \\
\hline 34 & 900 & $22^{\circ} 33^{\prime} 31.21 " \mathrm{~S}$ & $40^{\circ} 12^{\prime} 05.38^{\prime \prime} \mathrm{W}$ & $18 / 05 / 2002$ \\
\hline 36 & 1000 & $22^{\circ} 37^{\prime} 54.17^{\prime \prime} \mathrm{S}$ & $40^{\circ} 13^{\prime} 36.46^{\prime \prime} \mathrm{W}$ & $19 / 05 / 2002$ \\
\hline 37 & 1000 & $22^{\circ} 39^{\prime} 44.28^{\prime \prime} \mathrm{S}$ & $40^{\circ} 15^{\prime} 44.41^{\prime \prime} \mathrm{W}$ & $19 / 05 / 2002$ \\
\hline 38 & 1100 & $22^{\circ} 41^{\prime} 18.79^{\prime \prime} \mathrm{S}$ & $40^{\circ} 14^{\prime} 05.93 \mathrm{~W}$ & $15 / 05 / 2002$ \\
\hline 42 & 1200 & $22^{\circ} 41^{\prime} 39.45^{\prime \prime} \mathrm{S}$ & $40^{\circ} 10^{\prime} 24.84^{\prime \prime} \mathrm{W}$ & $15 / 05 / 2002$ \\
\hline 47 & 1650 & $22^{\circ} 11^{\prime} 04.40^{\prime \prime} \mathrm{S}$ & $39^{\circ} 47^{\prime} 04.60^{\prime \prime} \mathrm{W}$ & $25 / 11 / 2002$ \\
\hline 48 & 1950 & $22^{\circ} 11^{\prime} 16.63^{\prime \prime} \mathrm{S}$ & $39^{\circ} 43^{\prime} 44.70^{\prime \prime} \mathrm{W}$ & $25 / 11 / 2002$ \\
\hline 51 & 1350 & $22^{\circ} 04^{\prime} 43.44^{\prime \prime} \mathrm{S}$ & $39^{\circ} 49^{\prime} 08.29^{\prime \prime} \mathrm{W}$ & $24 / 11 / 2002$ \\
\hline 52 & 1650 & $22^{\circ} 04^{\prime} 44.26 " \mathrm{~S}$ & $39^{\circ} 46^{\prime} 31.55^{\prime \prime} \mathrm{W}$ & $24 / 11 / 2002$ \\
\hline 53 & 1950 & $22^{\circ} 04^{\prime} 46.20^{\prime \prime} \mathrm{S}$ & $39^{\circ} 43^{\prime} 02.02^{\prime \prime} \mathrm{W}$ & $24 / 11 / 2002$ \\
\hline 54 & 750 & $21^{\circ} 57^{\prime} 17.50^{\prime \prime} \mathrm{S}$ & $39^{\circ} 56^{\prime} 01.10^{\prime \prime} \mathrm{W}$ & $12 / 12 / 2002$ \\
\hline
\end{tabular}




\begin{tabular}{|c|c|c|c|c|}
\hline Station & Depth (m) & Latitude & Longitude & Date \\
\hline 57 & 1650 & $21^{\circ} 57^{\prime} 15.55^{\prime \prime} \mathrm{S}$ & $39^{\circ} 47^{\prime} 43.80^{\prime \prime W}$ & $14 / 12 / 2002$ \\
\hline 58 & 1950 & $21^{\circ} 57^{\prime} 26.87^{\prime \prime S}$ & $39^{\circ} 40^{\prime} 33.80^{\prime \prime W}$ & $11 / 12 / 2002$ \\
\hline 59 & 750 & $21^{\circ} 52^{\prime} 59.60^{\prime \prime} \mathrm{S}$ & $39^{\circ} 55^{\prime} 30.60^{\prime \prime W}$ & $12 / 12 / 2002$ \\
\hline 61 & 1350 & $21^{\circ} 52^{\prime} 51.90^{\prime \prime S}$ & $39^{\circ} 48^{\prime} 11.68^{\prime \prime W}$ & $12 / 12 / 2002$ \\
\hline 62 & 1650 & $21^{\circ} 52^{\prime} 41.91^{\prime \prime S}$ & $39^{\circ} 46^{\prime} 17.52^{\prime \prime W}$ & $11 / 12 / 2002$ \\
\hline 63 & 1950 & $21^{\circ} 52^{\prime} 44.10^{\prime \prime S}$ & $39^{\circ} 40^{\prime} 45.60^{\prime \prime W}$ & $11 / 12 / 2002$ \\
\hline 64 & 750 & $22^{\circ} 36^{\prime} 03.00^{\prime \prime} \mathrm{S}$ & $40^{\circ} 21^{\prime} 45.36^{\prime \prime W}$ & $22 / 11 / 2002$ \\
\hline 65 & 1050 & $22^{\circ} 40^{\prime} 57.81^{\prime \prime} \mathrm{S}$ & $40^{\circ} 16^{\prime} 30.35^{\prime \prime W}$ & $22 / 11 / 2002$ \\
\hline 68 & 1950 & $22^{\circ} 48^{\prime} 05.28^{\prime \prime S}$ & $40^{\circ} 06^{\prime} 38.64^{\prime \prime W}$ & $15 / 11 / 2002$ \\
\hline 69 & 750 & $22^{\circ} 31^{\prime} 12.47^{\prime \prime S}$ & $40^{\circ} 15^{\prime} 11.08^{\prime \prime W}$ & $22 / 11 / 2002$ \\
\hline 73 & 1950 & $22^{\circ} 41^{\prime} 35.24^{\prime \prime S}$ & $40^{\circ} 00^{\prime} 45.24^{\prime \prime W}$ & $22 / 11 / 2002$ \\
\hline 74 & 750 & $22^{\circ} 27^{\prime} 31.62^{\prime \prime S}$ & $40^{\circ} 09^{\prime} 23.19^{\prime \prime W}$ & $21 / 11 / 2002$ \\
\hline 75 & 1050 & $22^{\circ} 31^{\prime} 28.28^{\prime \prime} \mathrm{S}$ & $40^{\circ} 03^{\prime} 50.40^{\prime \prime W}$ & $19 / 11 / 2002$ \\
\hline 77 & 1650 & $22^{\circ} 36^{\prime} 03.37^{\prime \prime S}$ & $39^{\circ} 57^{\prime} 54.68^{\prime \prime W}$ & $16 / 11 / 2002$ \\
\hline 78 & 1950 & $22^{\circ} 37^{\prime} 02.47^{\prime \prime} \mathrm{S}$ & $39^{\circ} 56^{\prime} 20.52^{\prime \prime W}$ & $23 / 11 / 2002$ \\
\hline 81 & 1350 & $22^{\circ} 27^{\prime} 18.98^{\prime \prime S}$ & $39^{\circ} 54^{\prime} 50.48^{\prime \prime W}$ & $17 / 11 / 2002$ \\
\hline 83 & 1950 & $22^{\circ} 30^{\prime} 35.35^{\prime \prime} \mathrm{S}$ & $39^{\circ} 51^{\prime} 45.42^{\prime \prime W}$ & $23 / 11 / 2002$ \\
\hline 85 & 1350 & $22^{\circ} 29^{\prime} 33.89^{\prime \prime S}$ & $39^{\circ} 56^{\prime} 17.64 \mathrm{WW}$ & $19 / 11 / 2002$ \\
\hline 86 & 1650 & $22^{\circ} 31^{\prime} 36.00^{\prime \prime} \mathrm{S}$ & $39^{\circ} 55^{\prime} 15.00^{\prime \prime W}$ & $16 / 11 / 2002$ \\
\hline 87 & 1950 & $22^{\circ} 33^{\prime} 10.00^{\prime \prime S}$ & $39^{\circ} 54^{\prime} 22.00^{\prime W}$ & $23 / 11 / 2002$ \\
\hline 45 & 1050 & $22^{\circ} 10^{\prime} 53.40^{\prime \prime} \mathrm{S}$ & $39^{\circ} 52^{\prime} 18.30^{\prime \prime W}$ & $01 / 07 / 2003$ \\
\hline 46 & 1336 & $22^{\circ} 10^{\prime} 54.60^{\prime \prime S}$ & $39^{\circ} 48^{\prime} 59.50^{\prime \prime W}$ & $25 / 06 / 2003$ \\
\hline 48 & 1968 & $22^{\circ} 11^{\prime} 16.50^{\prime \prime S}$ & $39^{\circ} 43^{\prime} 44.60^{\prime \prime W}$ & $22 / 06 / 2003$ \\
\hline 49 & 722 & $22^{\circ} 04^{\prime} 32.80^{\prime \prime S}$ & $39^{\circ} 54^{\prime} 11.40^{\prime \prime W}$ & $30 / 06 / 2003$ \\
\hline 52 & 1643 & $22^{\circ} 04^{\prime} 45.20^{\prime \prime} \mathrm{S}$ & $39^{\circ} 46^{\prime} 31.70^{\prime \prime W}$ & $27 / 6 / 2003$ \\
\hline 53 & 1910 & $22^{\circ} 04^{\prime} 45.40^{\prime \prime S}$ & $39^{\circ} 41^{\prime} 58.50^{\prime \prime W}$ & $27 / 6 / 2003$ \\
\hline 54 & 698 & $21^{\circ} 57^{\prime} 11.80^{\prime \prime} \mathrm{S}$ & $39^{\circ} 56^{\prime} 04.20^{\prime \prime W}$ & $29 / 06 / 2003$ \\
\hline 56 & 1357 & $21^{\circ} 57^{\prime} 15.60^{\prime \prime S}$ & $39^{\circ} 49^{\prime} 37.50^{\prime \prime W}$ & $25 / 06 / 2003$ \\
\hline 58 & 1942 & $21^{\circ} 57^{\prime} 26.80^{\prime \prime} \mathrm{S}$ & $39^{\circ} 40^{\prime} 34.00^{\prime \prime W}$ & $27 / 06 / 2003$ \\
\hline 59 & 750 & $21^{\circ} 52^{\prime} 59.20^{\prime \prime} \mathrm{S}$ & $39^{\circ} 55^{\prime} 32.20^{\prime \prime W}$ & $29 / 06 / 2003$ \\
\hline 61 & 1350 & $21^{\circ} 52^{\prime} 51.80^{\prime \prime} \mathrm{S}$ & $39^{\circ} 48^{\prime} 12.50^{\prime \prime W}$ & $26 / 06 / 2003$ \\
\hline 63 & 1941 & $21^{\circ} 52^{\prime} 43.10^{\prime \prime} \mathrm{S}$ & $39^{\circ} 40^{\prime} 41.60^{\prime \prime W}$ & $26 / 06 / 2003$ \\
\hline 64 & 750 & $22^{\circ} 36^{\prime} 01.30^{\prime \prime S}$ & $40^{\circ} 21^{\prime} 43.70^{\prime \prime W}$ & $11 / 06 / 2003$ \\
\hline 65 & 1050 & $22^{\circ} 40^{\prime} 57.70 " \mathrm{~S}$ & $40^{\circ} 16^{\prime} 31.10^{\prime \prime W}$ & $11 / 06 / 2003$ \\
\hline 67 & 1596 & $22^{\circ} 46^{\prime} 58.30^{\prime \prime S}$ & $40^{\circ} 07^{\prime} 49.30^{\prime \prime W}$ & $12 / 06 / 2003$ \\
\hline 68 & 1972 & $22^{\circ} 48^{\prime} 05.90^{\prime \prime S}$ & $40^{\circ} 06^{\prime} 38.60^{\prime \prime W}$ & $12 / 06 / 2003$ \\
\hline 69 & 743 & $22^{\circ} 31^{\prime} 11.80^{\prime \prime S}$ & $40^{\circ} 15^{\prime} 12.10^{\prime \prime W}$ & $18 / 06 / 2003$ \\
\hline 71 & 1350 & $22^{\circ} 38^{\prime} 52.90^{\prime \prime S}$ & $40^{\circ} 04^{\prime} 16.30^{\prime \prime W}$ & $14 / 06 / 2003$ \\
\hline 75 & 1050 & $22^{\circ} 31^{\prime} 28.30^{\prime \prime S}$ & $40^{\circ} 03^{\prime} 49.30^{\prime \prime W}$ & $18 / 06 / 2003$ \\
\hline 77 & 1650 & $22^{\circ} 36^{\prime} 12.20^{\prime \prime S}$ & $39^{\circ} 58^{\prime} 22.90^{\prime \prime W}$ & $13 / 06 / 2003$ \\
\hline 78 & 1945 & $22^{\circ} 37^{\prime} 02.90 " \mathrm{~S}$ & $39^{\circ} 56^{\prime} 20.10^{\prime \prime W}$ & $13 / 06 / 2003$ \\
\hline 82 & 1650 & $22^{\circ} 28^{\prime} 46.50^{\prime \prime} \mathrm{S}$ & $39^{\circ} 53^{\prime} 27.90^{\prime \prime W}$ & $17 / 06 / 2003$ \\
\hline 84 & 1050 & $22^{\circ} 26^{\prime} 28.80^{\prime \prime S}$ & $39^{\circ} 58^{\prime} 53.30^{\prime \prime W}$ & $20 / 06 / 2003$ \\
\hline 86 & 1630 & $22^{\circ} 31^{\prime} 37.20^{\prime \prime S}$ & $39^{\circ} 55^{\prime} 14.50^{\prime \prime W}$ & $16 / 06 / 2003$ \\
\hline 87 & 1934 & $22^{\circ} 33^{\prime} 08.00^{\prime \prime S}$ & $39^{\circ} 54^{\prime} 21.50^{\prime \prime W}$ & $15 / 06 / 2003$ \\
\hline
\end{tabular}


Table 2. Table of the localities sampled as part of the Habitats Project - Campos Basin Environmental Heterogeneity.

\begin{tabular}{|c|c|c|c|c|c|c|}
\hline Cruise & Station & & Depth (m) & Latitude & Longitude & Date \\
\hline HAB 4 & D11 & R1 & 2449 & $22^{\circ} 52^{\prime} 15.30^{\prime \prime} \mathrm{S}$ & $40^{\circ} 05^{\prime} 10.40^{\prime \prime W}$ & $22 / 5 / 2008$ \\
\hline HAB 4 & G12 & R1 & 3236 & $22^{\circ} 12^{\prime} 19.50^{\prime \prime S}$ & $38^{\circ} 35^{\prime} 52.00^{\prime \prime W}$ & $25 / 5 / 2008$ \\
\hline HAB 6 & D07 & $\mathrm{R} 1$ & 698 & $22^{\circ} 36^{\prime} 27.10^{\prime \prime} \mathrm{S}$ & $40^{\circ} 22^{\prime} 29.60^{\prime \prime W}$ & $25 / 6 / 2008$ \\
\hline HAB 6 & D07 & $\mathrm{R} 2$ & 700 & $22^{\circ} 36^{\prime} 27.30^{\prime \prime} \mathrm{S}$ & $40^{\circ} 22^{\prime} 29.30^{\prime \prime W}$ & $25 / 6 / 2008$ \\
\hline HAB 6 & D07 & $\mathrm{R} 2$ & 700 & $22^{\circ} 36^{\prime} 27.30^{\prime \prime} \mathrm{S}$ & $40^{\circ} 22^{\prime} 29.30^{\prime \prime W}$ & $25 / 6 / 2008$ \\
\hline HAB 6 & A7 & R1 & 694 & $23^{\circ} 39^{\prime} 20.10^{\prime \prime S}$ & $41^{\circ} 18^{\prime} 30.30^{\prime \prime W}$ & $23 / 6 / 2008$ \\
\hline HAB 6 & A7 & $\mathrm{R} 2$ & 692 & $23^{\circ} 39^{\prime} 19.80^{\prime \prime} \mathrm{S}$ & $41^{\circ} 18^{\prime} 30.20^{\prime \prime W}$ & $23 / 6 / 2008$ \\
\hline HAB 6 & A7 & $\mathrm{R} 2$ & 692 & $23^{\circ} 39^{\prime} 19.80^{\prime \prime S}$ & $41^{\circ} 18^{\prime} 30.20^{\prime \prime W}$ & $23 / 6 / 2008$ \\
\hline HAB 6 & A7 & R3 & 733 & $23^{\circ} 39^{\prime} 19.90^{\prime \prime S}$ & $41^{\circ} 18^{\prime} 30.50^{\prime \prime W}$ & $24 / 6 / 2008$ \\
\hline HAB 6 & CANAC7 & $\mathrm{R} 1$ & 758 & $21^{\circ} 47^{\prime} 26.70^{\prime \prime S}$ & $40^{\circ} 02^{\prime} 13.30^{\prime \prime} \mathrm{W}$ & $28 / 6 / 2008$ \\
\hline HAB 6 & CANAC7 & $\mathrm{R} 2$ & 753 & $21^{\circ} 47^{\prime} 26.60^{\prime \prime} \mathrm{S}$ & $40^{\circ} 02^{\prime} 13.70^{\prime \prime W}$ & $28 / 6 / 2008$ \\
\hline HAB 6 & I07 & R1 & 694 & $21^{\circ} 11^{\prime} 12.20^{\prime \prime S}$ & $40^{\circ} 12^{\prime} 52.00^{\prime \prime W}$ & $29 / 6 / 2008$ \\
\hline HAB 6 & D07 & $\mathrm{R} 2$ & 700 & $22^{\circ} 36^{\prime} 27.30^{\prime \prime S}$ & $40^{\circ} 22^{\prime} 29.00^{\prime \prime W}$ & $25 / 6 / 2008$ \\
\hline HAB 6 & D07 & $\mathrm{R} 2$ & 700 & $22^{\circ} 36^{\prime} 27.30^{\prime \prime} \mathrm{S}$ & $40^{\circ} 22^{\prime} 29.30^{\prime \prime} \mathrm{W}$ & $25 / 6 / 2008$ \\
\hline HAB 6 & D07 & R1 & 698 & $22^{\circ} 36^{\prime} 27.10^{\prime \prime} \mathrm{S}$ & $40^{\circ} 22^{\prime} 29.60^{\prime \prime W}$ & $25 / 6 / 2008$ \\
\hline HAB 6 & D07 & $\mathrm{R} 2$ & 700 & $22^{\circ} 36^{\prime} 27.30^{\prime \prime} \mathrm{S}$ & $40^{\circ} 22^{\prime} 29.30^{\prime \prime W}$ & $25 / 6 / 2008$ \\
\hline HAB 7 & D06 & R1 & 396 & $22^{\circ} 33^{\prime} 35.70^{\prime \prime} \mathrm{S}$ & $40^{\circ} 26^{\prime} 38.90^{\prime \prime W}$ & $08 / 7 / 2008$ \\
\hline HAB 7 & D06 & R3 & 393 & $22^{\circ} 33^{\prime} 33.80^{\prime \prime} \mathrm{S}$ & $40^{\circ} 26^{\prime} 40.30^{\prime \prime W}$ & $11 / 7 / 2008$ \\
\hline HAB 7 & $\mathrm{H} 7$ & R1 & 700 & $21^{\circ} 41^{\prime} 12.30^{\prime \prime S}$ & $40^{\circ} 02^{\prime} 20.20^{\prime \prime W}$ & $07 / 7 / 2008$ \\
\hline HAB 7 & $\mathrm{H} 7$ & $\mathrm{R} 2$ & 699 & $21^{\circ} 41^{\prime} 11.70^{\prime \prime S}$ & $40^{\circ} 02^{\prime} 20.70^{\prime \prime W}$ & $07 / 7 / 2008$ \\
\hline HAB 7 & $\mathrm{H} 7$ & R3 & 700 & $21^{\circ} 41^{\prime} 11.80^{\prime \prime S}$ & $40^{\circ} 02^{\prime} 20.40^{\prime \prime W}$ & $07 / 7 / 2008$ \\
\hline HAB 7 & I07 & R3 & 792 & $21^{\circ} 11^{\prime} 02.60^{\prime \prime} \mathrm{S}$ & $40^{\circ} 12^{\prime} 18.20^{\prime \prime W}$ & $05 / 7 / 2008$ \\
\hline HAB 8 & D06 & $\mathrm{R} 2$ & 401 & $22^{\circ} 33^{\prime} 35.10^{\prime \prime S}$ & $40^{\circ} 26^{\prime} 37.50^{\prime \prime W}$ & $31 / 1 / 2009$ \\
\hline HAB 8 & D07 & $\mathrm{R} 2$ & 696 & $22^{\circ} 36^{\prime} 25.30^{\prime \prime} \mathrm{S}$ & $40^{\circ} 22^{\prime} 30.60^{\prime \prime W}$ & $29 / 1 / 2009$ \\
\hline HAB 8 & $\mathrm{C} 10$ & R3 & 1953 & $23^{\circ} 08^{\prime} 23.80^{\prime \prime} \mathrm{S}$ & $40^{\circ} 36^{\prime} 37.90^{\prime \prime W}$ & $27 / 1 / 2009$ \\
\hline HAB 8 & A07 & $\mathrm{R} 2$ & 701 & $23^{\circ} 39^{\prime} 20.60^{\prime \prime S}$ & $41^{\circ} 18^{\prime} 28.20^{\prime \prime W}$ & $28 / 1 / 2009$ \\
\hline HAB 8 & A07 & R3 & 693 & $23^{\circ} 39^{\prime} 21.90^{\prime \prime} \mathrm{S}$ & $41^{\circ} 18^{\prime} 33.10^{\prime \prime W}$ & $28 / 1 / 2009$ \\
\hline HAB 9 & CANAC7 & $\mathrm{R} 2$ & 780 & $21^{\circ} 47^{\prime} 26.60^{\prime \prime} \mathrm{S}$ & $40^{\circ} 01^{\prime} 55.30^{\prime \prime W}$ & $06 / 2 / 2009$ \\
\hline HAB 9 & CANAC7 & R3 & 775 & $21^{\circ} 47^{\prime} 26.70^{\prime \prime S}$ & $40^{\circ} 01^{\prime} 55.50^{\prime \prime W}$ & $06 / 2 / 2009$ \\
\hline HAB 9 & CANG-7 & $\mathrm{R} 2$ & 720 & $21^{\circ} 56^{\prime} 11.90^{\prime \prime S}$ & $39^{\circ} 57^{\prime} 45.30^{\prime \prime W}$ & $07 / 2 / 2009$ \\
\hline HAB 9 & $\mathrm{H} 07$ & $\mathrm{R} 2$ & 702 & $21^{\circ} 41^{\prime} 12.60^{\prime \prime S}$ & $40^{\circ} 01^{\prime} 56.10^{\prime \prime} \mathrm{W}$ & $06 / 2 / 2009$ \\
\hline
\end{tabular}

ger teeth. The width of the hinge plate was measured in the thicker part with a straight line (Figs 1-2).

The material analyzed in this study is deposited in the Mollusca collections of the following institutions: Departamento de Zoologia, Instituto de Biologia, Universidade Federal do Rio de Janeiro (IBUFRJ); Museu Nacional do Rio de Janeiro (MNRJ); Museu de Zoologia da Universidade de São Paulo (MZUSP); Museu Oceanográfico da Fundação Universitária de Rio Grande (MOFURG); National Museum of Natural History, Smithsonian Institute (USNM); and Muséum National d'Histoire Naturelle, Paris (MNHN). The following abbreviations are used: \# - station; IBUFRJ - Instituto 

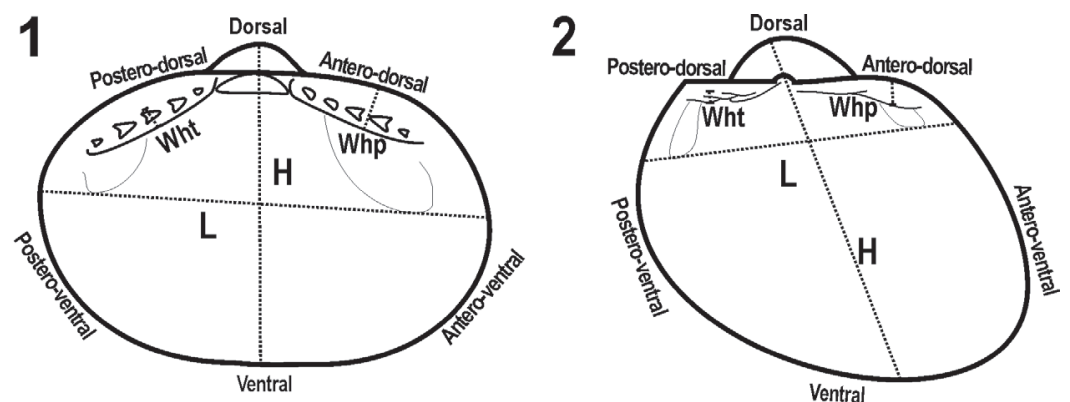

Figures I-2. Scheme of the measurements and abbreviations used in the descriptions of the species A Microgloma $\mathbf{B}$ Pristigloma $\mathbf{H}$ height $\mathbf{L}$ length, wht - width of hinge teeth, whp - width of hinge plate.

de Biologia da Universidade Federal do Rio de Janeiro; MNRJ - Museu Nacional do Rio de Janeiro; MZUSP - Museu de Zoologia da Universidade de São Paulo; MOFURG - Museu Oceanográfico da Fundação Universitária de Rio Grande; MNHN Muséum National d'Histoire Naturelle, Paris; USNM - National Museum of Natural History, Smithsonian Institute; MCZ - Museum of Comparative Zoology, Harvard University, Cambridge, U.S.A.

\section{Systematics}

Pristigloma alba Sanders \& Allen, 1973 http://species-id.net/wiki/Pristigloma_alba Figs 3-8

Pristigloma alba Sanders \& Allen, 1973: 245, fig 5; Allen 2008: 67, 87, 95, 97-101, $103,111,113,119,141,146,152,153,157,167,168,173$. Oliver et al. 2009: figs. MO11691-11697 (available online).

Type specimen. MCZ 271976. We tried to find this lot in the MCZ with the help of Mr. Cleo Oliveira, but the curators could not find it. We made contact with Dr John A. Allen who sent us live specimens from Surinam Basin \#293 (08 $58^{\prime}$ N5 $54^{\circ} 04^{\prime} \mathrm{W}$, $1518 \mathrm{~m}$ ) to compare with Campos Basin specimens. Furthermore, using the good description in Sanders and Allen (1973) and figures of the specimens of Rockall Trough and Biscay Bay available in Oliver et al. (2009) we could properly identify our specimens.

Geographical distribution. Angola, 3739-4597 m; Canaries, 6709-6711 m; North America, 2178-4892 m; Brazil, 3459 m; Argentina, 4382-4405 m (all from Sanders and Allen 1973); Cape Verde, 3495 m; Angola, 3797 m; Canaries, 2351-3000 m; West Europe, 2897-4660 m; Newfoundland, 4400 m; North America, 2178-4833 m; Surinam, 5100 m; Brazil, 3495 m; Argentine, 4402 m (all from Allen 2008); Rock- 


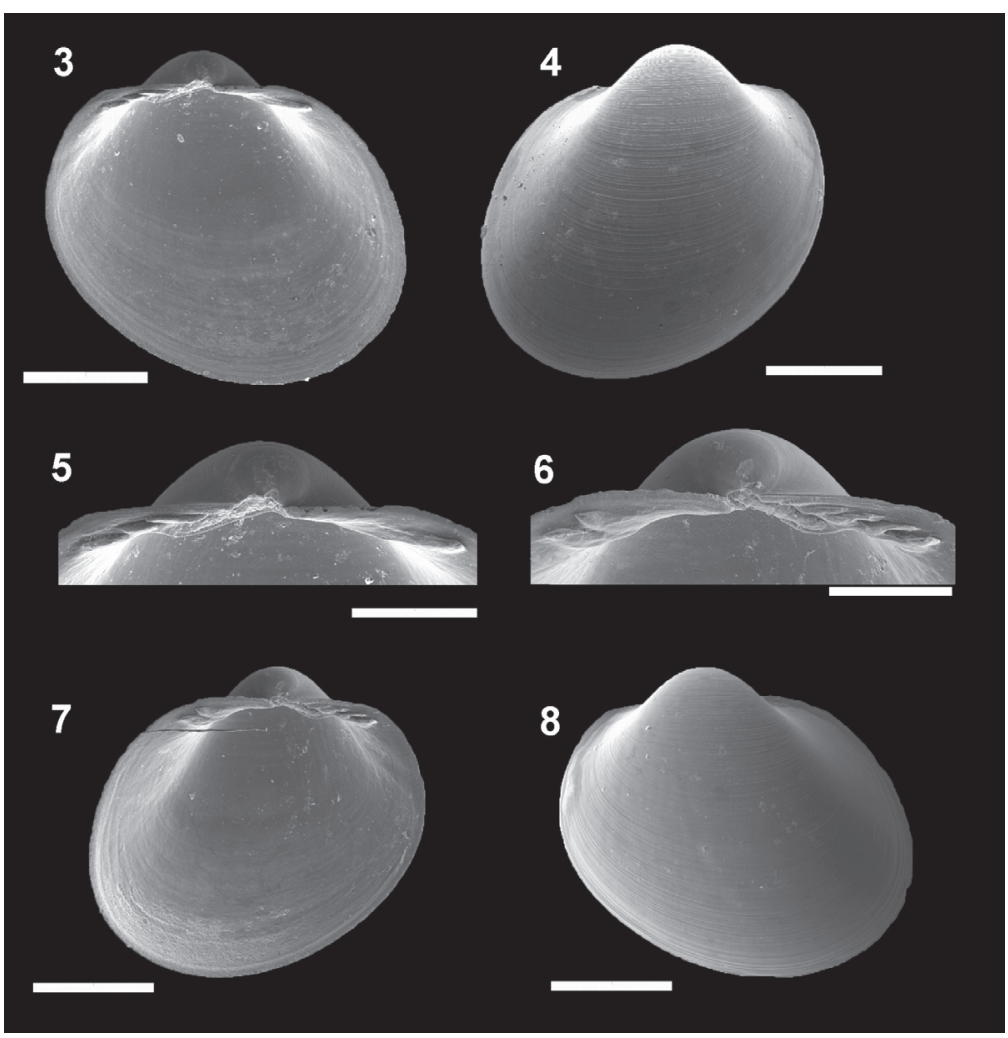

Figure 3-8. Pristigloma alba Sanders \& Allen, 1973 IBUFRJ 16161. Left valve: internal view 3 external view $\mathbf{4}$ detail of the hinge plate $\mathbf{5}$ Right valve: detail of the hinge plate $\mathbf{6}$ internal view $\mathbf{7}$ external view 8 Scale bars 3-4, 7-8 = 500 $\mu \mathrm{m}, 5-6=250 \mu \mathrm{m}$

all Trough and Biscay Bay mostly in depths over 2000 m (Oliver et al. 2009); BrazilCampos Basin, 1200-1972 m (present study).

Material examined. MNRJ 19114 (\# 68, 2003), 4 valves; MZUSP 99977 (\# 68, 2003), 4 valves; IBUFRJ 16161 (\# 68, 2002), 3 valves; IBUFRJ 19001 (\# 10, 2002), 5 valves; IBUFRJ 19002 (\# 42, 2002), 3 valves; IBUFRJ 19003 (\# 47, 2002), 1 valves; IBUFRJ 19004 (\# 48, 2002), 7 valves; IBUFRJ 19005 (\# 52, 2002), 1 valve; IBUFRJ 19006 (\# 58, 2002), 6 valves; IBUFRJ 19007 (\# 62, 2002), 3 valves; IBUFRJ 19008 (\# 63, 2002), 6 valves; IBUFRJ 19009 (\# 73, 2002), 14 valves; IBUFRJ 19010 (\# 77, 2002), 2 valves; IBUFRJ 19011 (\# 78, 2002), 4 valves; IBUFRJ 19012 (\# 83, 2002), 2 valves; IBUFRJ 19013 (\# 87, 2002), 6 valves; IBUFRJ 19014 (\# 46, 2003), 1 valve; IBUFRJ 19015 (\# 48, 2003), 15 valves; IBUFRJ 19016 (\# 52, 2003), 1 valve; IBUFRJ 19017 (\# 53, 2003), 16 valves; IBUFRJ 19018 (\# 58, 2003), 3 valves; IBUFRJ 19019 (\# 61, 2003), 2 valves; IBUFRJ 19020 (\# 63, 2003), 16 valves; IBUFRJ 19021 (\# 68, 2003), 14 valves; IBUFRJ 19022 (\# 72, 2003), 1 valve; IBUFRJ 19023 (\# 73, 2003), 8 valves; IBUFRJ 19024 
(\# 78, 2003), 14 valves; IBUFRJ 19025 (\# 82, 2003), 1 valve; IBUFRJ 19026 (\# 86, 2003), 4 valves.

Characterization. Shell H/L ratio about 1.04 . W/H ratio approximately 0.86 . Muscle scars rarely visible; anterior adductor scar $2 / 3$ of posterior scar. Anterior and posterior parts of the hinge plate usually of same length. Width of posterior row of teeth occupies about $65 \%$ of width of hinge plate, which is thin for its size $(\mathrm{whp} / \mathrm{H}$ approximately 0.07). Posterior part of the hinge plate longer than anterior one. Prodissoconch smooth, with length approximately $120 \mu \mathrm{m}$.

Remarks. This species was recently well described and for this reason we add only new information on the proportions of the shell (H/L and width) and hinge plate characteristics. Sanders and Allen (1973) noted no evident muscle scars or pallial line. In the Campos Basin specimens, no pallial line is discernible on the valves, but faint muscle scars are apparent in some specimens. It is a common species in the Atlantic, and its occurrence in the Campos Basin was expected, since it was previously recorded from the northern Brazilian coast (Pernambuco) and from Argentina. The present study provides new points to the geographical distribution of $P$. alba, and is also the shallowest $(1200 \mathrm{~m})$ record for the species, which was previously known only from abyssal depths (2100-4898 m).

\section{Microgloma mirmidina (Dautzenberg \& Fischer, 1897)}

http://species-id.net/wiki/Microgloma_mirmidina

Figs $9-13$

Leda mirmidina Dautzenberg and Fischer, 1897: 208, pl. 6, figs 11-14; Dautzenberg 1927: 292, pl. 8, figs 27-30.

Nuculana mirmidina: Clarke, 1962: 53.

Microgloma mirmidina: La Perna, 2008: 154, 155, fig 6.

Geographical distribution. Southeast of Flores, Azores, 1846 m (La Perna, 2008). Campos Basin 1050-1950 m (present study).

Material examined. MNRJ 19115 (\#68, 2003), 6 valves; MZSP 99978 (\#71, 2003), 7 valves; IBUFRJ 15889 (\#67, 2003), 5 valves; IBUFRJ 17501 (\#87, 2003), 4 valves; IBUFRJ 19084 (\# 10, 2001), 4 valves; IBUFRJ 19085 (\# 28, 2001), 1 valve; IBUFRJ 19086 (\# 42, 2002), 1 valve; IBUFRJ 19087 (\# 48, 2002), 6 valves and 2 specimens; IBUFRJ 19088 (\# 51, 2002), 1 valve; IBUFRJ 19089 (\# 53, 2002), 6 valves and 1 specimen; IBUFRJ 19090 (\# 57, 2002), 1 valve IBUFRJ 19091 (\# 62, 2002), 4 valves; IBUFRJ 19092 (\# 63, 2002), 10 valves; IBUFRJ 19093 (\# 68, 2002), 1 valve; IBUFRJ 19094 (\# 73, 2002), 6 valves and 1 specimen; IBUFRJ 19095 (\# 75, 2002), 1 valve; IBUFRJ 19096 (\# 77, 2002), 12 valves and 1 specimen; IBUFRJ 19097 (\# 78, 2002), 4 valves; IBUFRJ 19098 (\# 81, 2002), 2 valves; IBUFRJ 19098 (\# 81, 2002), 2 valves; IBUFRJ 19099 (\# 83, 2002), 4 valves 


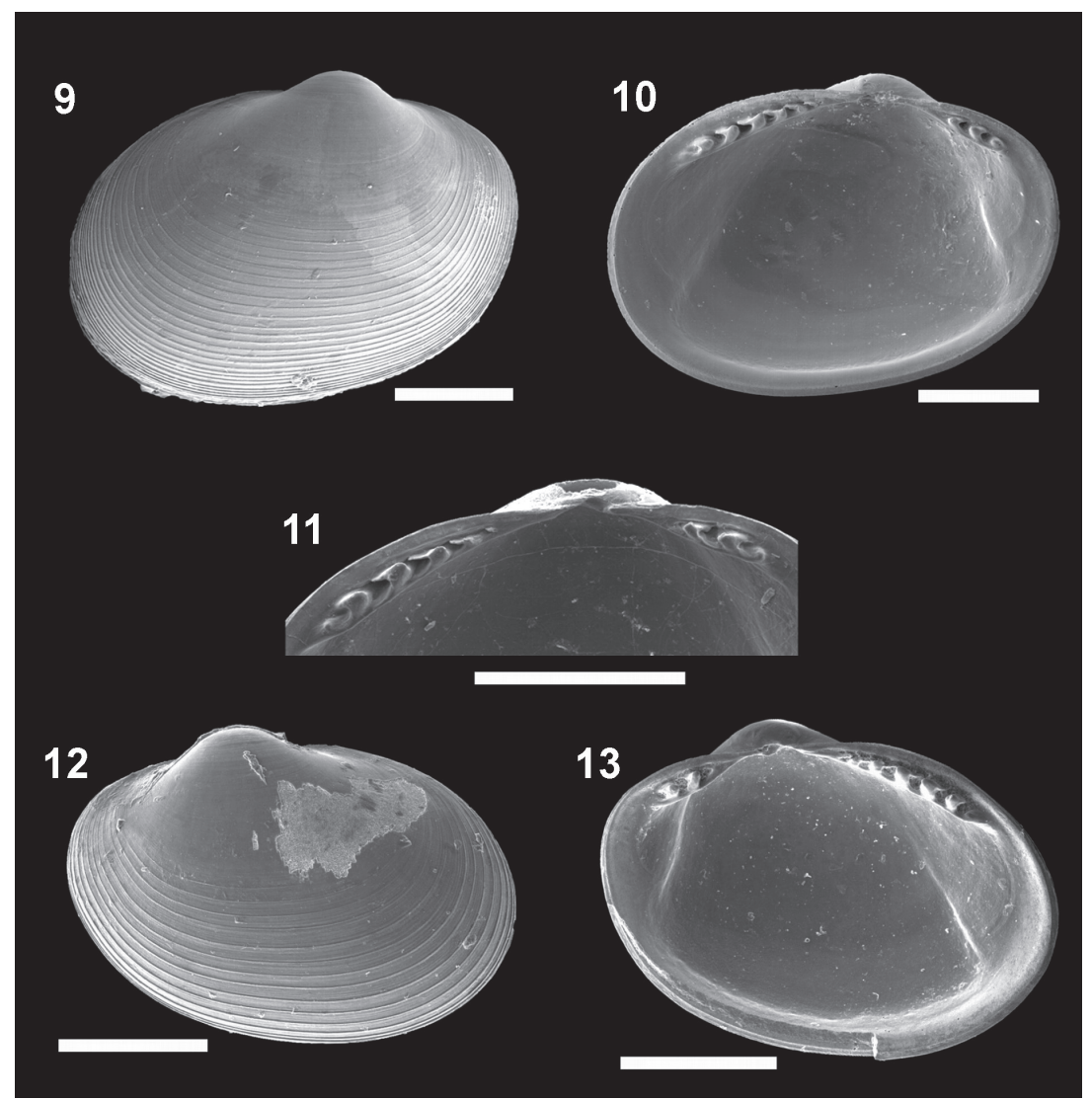

Figures 9-13. Microgloma mirmidina (Dautzenberg \& Fischer, 1897). External view, left valve 9 internal view, right valve $\mathbf{I} \mathbf{0}$ Detail of the hinge plate II External view, right valve $\mathbf{I} \mathbf{2}$ internal view, left valve 13 All from the lot IBUFRJ 15889. Scale bars $A=200 \mu \mathrm{m} ; B, E=300 \mu \mathrm{m} ; C=100 \mu \mathrm{m} ; D=250 \mu \mathrm{m}$.

and 1 specimen; IBUFRJ 19100 (\# 85, 2002), 1 valve; IBUFRJ 19101 (\# 86, 2002), 6 valves; IBUFRJ 19102 (\# 87, 2002), 5 valves; IBUFRJ 19103 (\# 50A, 2003), 1 valve; IBUFRJ 19105 (\# 61, 2003), 3 valves; IBUFRJ 19106 (\# 63, 2003), 2 valves; IBUFRJ 19107 (\# 72, 2003), 2 valves and 1 specimen; IBUFRJ 19108 (\# 73, 2003), 4 valves; IBUFRJ 19109 (\# 77, 2003), 5 valves and 3 specimens; IBUFRJ 19110 (\# 78, 2003), 4 valves; IBUFRJ 19111 (\# 82, 2003), 2 valves; IBUFRJ 19112 (\# 84, 2003), 2 valves; IBUFRJ 19113 (\# 86, 2003), 2 valves; IBUFRJ 19114 (\# C10, 2008), 1 valve.

Characterization. Shell H/L approximately 0.79 (n 10, min 0.75, max 0.83), W/H approx. 0.59(n 10, min 0.53, max 0.63). Posterior part of the hinge plate shorter than anterior one. Width of both the anterior and posterior rows of teeth occupies nearly half of the width of the hinge plate, which is moderately thick for its size (whp/H about 0.11 ). Prodissoconch smooth, length approximately $176 \mu \mathrm{m}$. 
Remarks. The radial sculpture on the prodissoconch surface is absent in $M$. mirmidina, but admittedly it is not always clearly developed in the other species of Microgloma (La Perna 2008). Microgloma mirmidina differs from other species of the genus in the elongated outline, more inflated shells and small hinge plate. In relation to the type material of $M$. mirmidina figured by La Perna (2008) the Campos Basin material presents a smaller hinge plate. The figured specimens presents $\mathrm{H} / \mathrm{L}$ ratio of $0.72,0.76$ and 0.83 (La Perna 2008 fig 6 B, E, I, respectively). Despite geographical distance between the two records of the present species, these conchological differences are not pronounced enough to affirm they belong to distinct species.

\section{Microgloma macaron sp. $\mathbf{n}$.}

urn:lsid:zoobank.org:act:31275EB9-546D-414B-82B0-C59CA55F8C93

http://species-id.net/wiki/Microgloma_macaron

Figs $14-22$

Holotype. MNRJ 19.112 (Figs 14, 16, 18).

Type locality. Campos Basin, \#54, 12/12/2002, 750m, 21 ${ }^{\circ} 57^{\prime} 17,5^{\prime \prime S}, 39^{\circ} 56^{\prime}$ 01,1"W.

Paratypes. IBUFRJ 15297, 8 valves and 2 specimens; MZUSP 99979 , 4 valves; USNM 1156943 , 6 valves; MNHN 24596, 6 valves; all from the type locality.

Etymology. The species epithet refers to the French macaroon cookie ("macaron" in the French language), which has a similar appearance to the articulated valves. The species epithet is proposed as a noun in apposition.

Material examined. IBUFRJ 19145 (\# 32, 2002), 3 valves; IBUFRJ 19146 (\# 33, 2002), 1 valve; IBUFRJ 19147 (\# 34, 2002), 1 valve; IBUFRJ 19148 (\# 36, 2002), 1 valve; IBUFRJ 15482 (\# 54, 2002), 6 valves; IBUFRJ 17033 (\# 59, 2002), 1 specimen; IBUFRJ 19150 (\# 61, 2002), 3 specimens; IBUFRJ 16074 (\# 64, 2002), 14 valves and 1 specimen; IBUFRJ 15141 (\# 69, 2002), 7 valves; IBUFRJ 15285 (\# 74, 2002), 2 valves; IBUFRJ 15635 (\# 54, 2003), 32 valves and 5 specimens; IBUFRJ 19152 (\# 59, 2003), 6 valves and 1 specimen; IBUFRJ 19153 (\# 61, 2003), 1 valve; IBUFRJ 19153 (\# 61, 2003), 1 valve; IBUFRJ 19154 (\# 64, 2003), 18 valves and 4 specimens; IBUFRJ 19155 (\# 69, 2003), 4 valves; IBUFRJ 19156 (\# D11, 2008), 1 specimen; IBUFRJ 19157 (\# G12, 2008), 1 specimen; IBUFRJ 19158 (\# A7, 2008), 10 specimens; IBUFRJ 19159 (\# A7, 2008), 1 specimen; IBUFRJ 19160 (\# A7, 2008), 2 valves and 5 specimens; IBUFRJ 19161 (\# D7, 2008), 1 specimen; IBUFRJ 19162 (\# D7, 2008), 6 specimens; IBUFRJ 19163 (\# CANAC7, 2008), 1 specimen; IBUFRJ 19164 (\# H7, 2008), 1 specimen; IBUFRJ 19165 (\# H7, 2008), 1 specimen; IBUFRJ 19166 (\# H7, 2008), 5 specimens; IBUFRJ 19167 (\# I7, 2008), 2 specimens; IBUFRJ 19168 (\# A7, 2009), 3 specimens; IBUFRJ 19169 (\# A7, 2009), 5 specimens; IBUFRJ 19170 (\# D6, 2009), 1 specimen; IBUFRJ 19171 (\# D7, 2009), 1 specimen; IBUFRJ 19172 (\# H7, 2009), 12 specimens; IBUFRJ 19173 (\# CANAC7, 2009), 1 specimen; IBUFRJ 
19174 (\# CANAC7, 2009), 1 specimen; IBUFRJ 19175 (\# CANG7, 2009), 3 specimens; IBUFRJ 19177 (\# 64, 2003), 4 specimens.

Diagnosis. Shell minute, ovate. Entire shell margin thickened and covered by an extension of the periostracum. Prodissoconch surface with one subtle radial striae.

Description. Shell minute, ovate, H/L ratio about 0.81 (n 38, min 0.75 , max 0.92 ), $\mathrm{W} / \mathrm{H}$ ratio about 0.84 ( $\mathrm{n} 14$, min 0.77 , max 0.87 ), glossy, translucent, robust for its size, equilateral; umbones prominent, large, posterior to midline, orthogyrous. Antero-dorsal margin straight, oblique; anterior margin rounded, extended. Antero-ventral margin, postero-ventral margin shorter and rising up to the short posterior end; posterior margin slightly truncated, forming a small shoulder. Entire shell margin thickened and covered by an extension of the periostracum, resembling a macaroon in ventral view (Figs 21-22). Surface with commarginal growth lines. Hinge plate with 5-7 anterior and 4-5 posterior teeth, interrupted by a large, rectangular and shallow resilifer. Width of both anterior and posterior row of teeth occupies about $70 \%$ of width of hinge plate, which is thick for its size (whp/ $\mathrm{H}$ approximately Campos Basin, \#54, 12/12/2002 0.14). Posterior part of the hinge plate shorter than anterior one. Prodissoconch surface nacreous, with one subtle radial striae (Fig. 20), and length approximately $244 \mu \mathrm{m}$. Maximum adult shell length $1.20 \mathrm{~mm}$.

Remarks. In some other species of Microgloma, the shell is expanded ventrally, around the valve, which enlarges the internal volume of the animal and counterbal-

Table 3. Measurements of the type material. ${ }^{*}$ Width $=$ width of a single valve.

\begin{tabular}{l|c|c|c}
\hline \multicolumn{4}{|c}{ M. macaron sp. n. } \\
\hline & Length & Height & Width \\
\hline MNRJ 19.112 & 1.20 & 1.00 & 0.36 \\
\hline IBUFRJ 15.297 & 1.11 & 0.87 & 0,31 \\
\hline IBUFRJ 15.297 & 1.11 & 0.89 & 0.36 \\
\hline IBUFRJ 15.297 & 1.11 & 0.91 & 0.36 \\
\hline IBUFRJ 15.297 & 1.11 & 0.84 & 0.33 \\
\hline IBUFRJ 15.297 & 1.11 & 0.89 & 0.36 \\
\hline IBUFRJ 15.297 & 1.11 & 0.89 & 0.36 \\
\hline IBUFRJ 15.297 & 1.16 & 0.93 & 0.38 \\
\hline IBUFRJ 15.297 & 1.11 & 0.91 & 0.33 \\
\hline IBUFRJ 15.297 & 1.13 & 0.82 & 0.31 \\
\hline IBUFRJ 15.297 & 1.13 & 0.89 & 0.33 \\
\hline MZSP 99.979 & 1.11 & 0.87 & 0.33 \\
\hline MZSP 99.979 & 1.13 & 1.04 & 0.36 \\
\hline MZSP 99.979 & 1.16 & 0.93 & 0.38 \\
\hline MZSP 99.979 & 1.11 & 0.91 & 0.31 \\
\hline USNM 1156943 & 1.11 & 0.89 & 0.36 \\
\hline USNM 1156943 & 1.11 & 0.89 & 0.36 \\
\hline USNM 1156943 & 1.13 & 0.91 & 0.36 \\
\hline USNM 1156943 & 1.13 & 0.89 & 0.38 \\
\hline USNM 1156943 & 1.13 & 0.91 & 0.33 \\
\hline
\end{tabular}




\begin{tabular}{l|c|c|c}
\hline \multicolumn{4}{|c}{ M. macaron sp. n. } \\
\hline & Length & Height & Width* \\
\hline USNM 1156943 & 1.11 & 0.89 & 0.36 \\
\hline MNHN 24596 & 1.11 & 0.91 & 0.33 \\
\hline MNHN 24596 & 1.18 & 0.93 & 0.38 \\
\hline MNHN 24596 & 1.11 & 0.91 & 0.36 \\
\hline MNHN 24596 & 1.11 & 0.89 & 0.38 \\
\hline MNHN 24596 & 1.11 & 0.91 & 0.36 \\
\hline MNHN 24596 & 1.04 & 0.87 & 0.38 \\
\hline
\end{tabular}

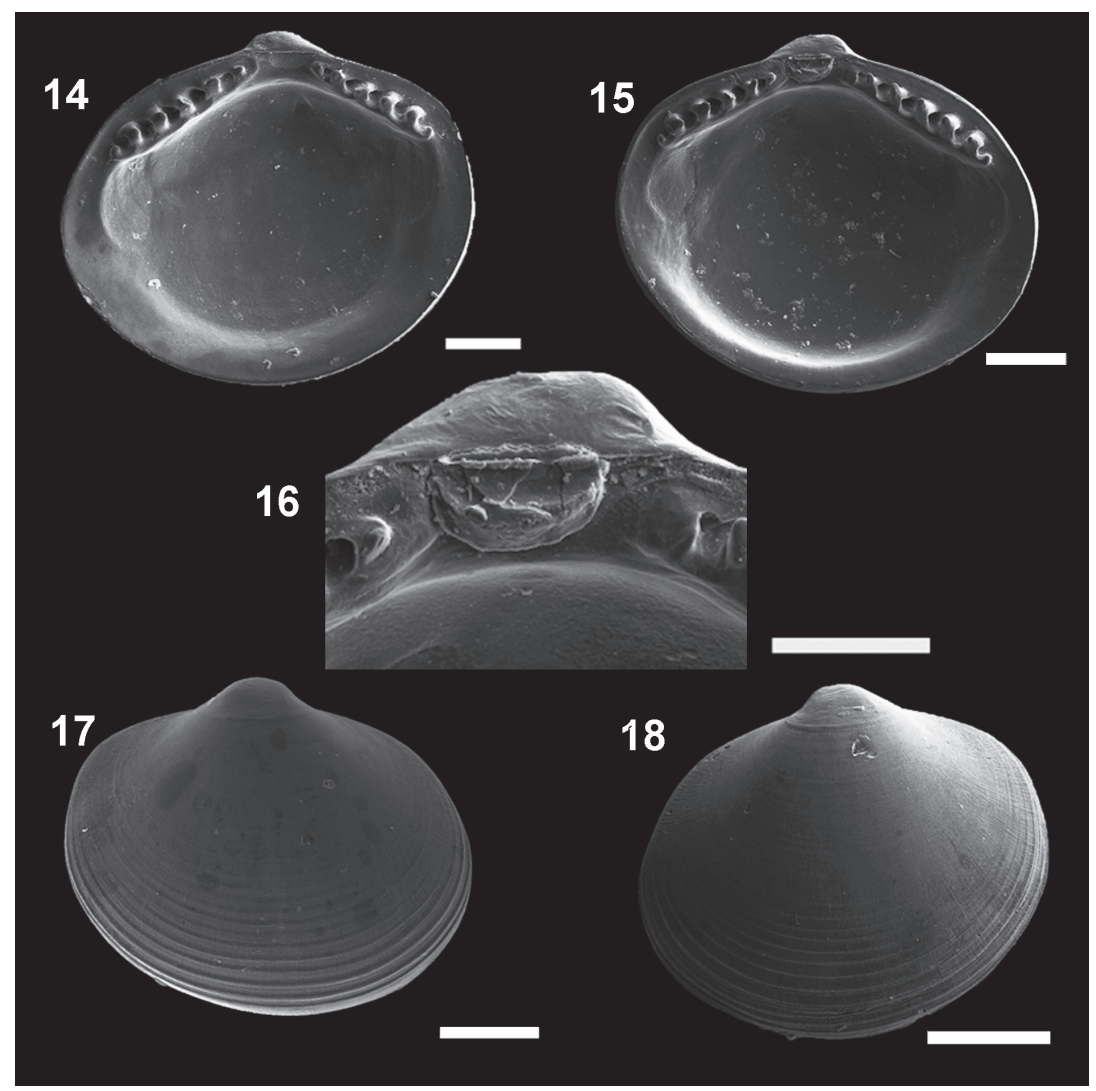

Figures 14-18. Microgloma macaron sp. n. Internal view, right valve $\mathbf{4}$ left valve $\mathbf{I} \mathbf{5}$ detail of the hinge plate and ligament $\mathbf{I} 6$ External view, right valve $I 7$ left valve $I 8$ Holotype MNRJ $19112(14,16,18)$. Paratype IBUFRJ 15297 I5, I7 Scale bars: 14, 16= $200 \mu \mathrm{m} ; 15,18=300 \mu \mathrm{m} ; 17=250 \mu \mathrm{m}$.

ances the effects of miniaturization (Ockelmann and Warén 1998, La Perna 2008). However, in $M$. macaron this expansion is thicker, and the periostracum surrounds the entire margin (Fig 22). This characteristic of the periostracum deserves special attention since it not only covers the shell to the margins in the usual way, but is more conspicuous in this area, giving the impression that valves do not articulate. We are 


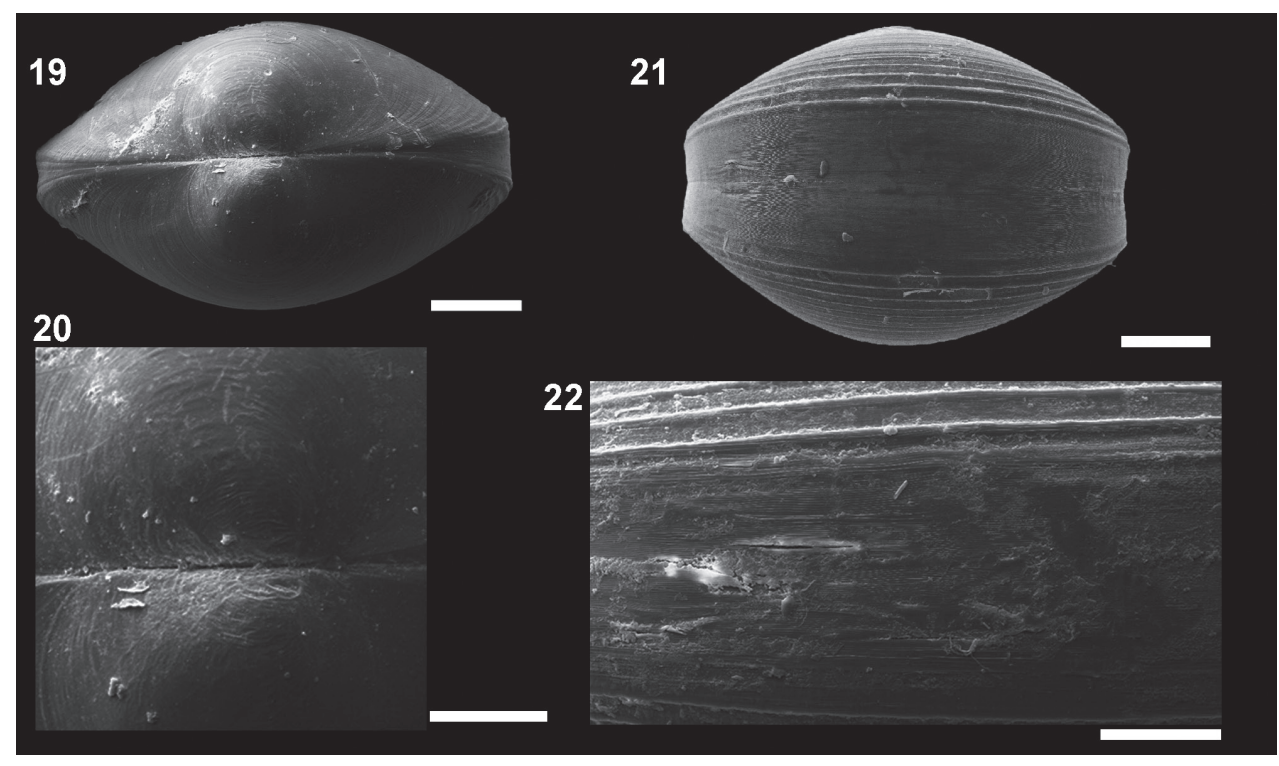

Figures 19-22. Microgloma macaron sp. n. Dorsal view 19 prodissoconch 20 (IBUFRJ 15297). Ventral margin view, extended margin $\mathbf{2} \mathbf{I}$ periostracum of the ventral margin 22 (IBUFRJ 19177). Scale bars: $\mathbf{I 9 , 2} \mathbf{I}=200 \mu \mathrm{m} ; \mathbf{2 0}, \mathbf{2 2}=100 \mu \mathrm{m}$.

not sure how this system works, and how the animal, in spite of having these fibers surrounding the valve apertures, can have water flux in the mantle cavity.

Compared to $M$. pusilla and $M$. mirmidina, $M$. macaron is distinguished by the ovate outline, umbones at midline and much more projecting, and a thicker hinge plate. Microgloma macaron is similar to $M$. yongei in outline, but compared with the paratypes figured by Ockelmann and Warén (1998, page 14, fig 6-D), the former has more prominent umbones, a thicker hinge plate, as well as a larger resilifer. $M i$ crogloma macaron can be distinguished from $M$. tumidula by the shape of the teeth, which are not as inclined as in this latter species. The anterior and posterior areas of the hinge plate form a less obtuse angle compared to those of $M$. tumidula.

Except for two, probably worn, valves found at two stations at approx. $3000 \mathrm{~m}$ depth, and one at $1970 \mathrm{~m}$, this species is concentrated at depths between 400-750 m. Untill now this species is reccorded solely in Campos Basin.

\section{Microgloma nhanduti sp. n.}

urn:lsid:zoobank.org:act:511F7840-3490-4EA1-A48F-CB56C60329FC http://species-id.net/wiki/Microgloma_nhanduti

Figs 23-31

Holotype. MNRJ 19.113 (Figs 26, 28).

Type locality. Campos Basin, \#54, 12/12/2002, 750m, 21 $57^{\prime} 17,5^{\prime S}$, 39 56'01,1"W. 
Table 4. Measurements of the type material. ${ }^{*}$ Width $=$ width of a single valve.

\begin{tabular}{l|c|c|c}
\hline \multicolumn{5}{|c}{ M. nhanduti sp. $\boldsymbol{n}$. } \\
\hline & Length & Height & Width \\
\hline MNRJ 19.113 & 1.12 & 0.90 & 0.29 \\
\hline IBUFRJ 14.991 & 1.11 & 0.84 & 0.24 \\
\hline IBUFRJ 14.991 & 1.04 & 0.82 & 0.29 \\
\hline IBUFRJ 14.991 & 1.09 & 0.89 & 0.29 \\
\hline IBUFRJ 19.176 & 1.13 & 0.89 & 0.29 \\
\hline MZSP 99.980 & 1.09 & 0.82 & 0.29 \\
\hline MZSP 99.980 & 1.09 & 0.82 & 0.27 \\
\hline MZSP 99.980 & 0.98 & 0.76 & 0.24 \\
\hline MZSP 99.980 & 1.09 & 0.82 & 0.27 \\
\hline MZSP 99.980 & 1.11 & 0.82 & 0.27 \\
\hline MZSP 99.980 & 1.11 & 0.84 & 0.22 \\
\hline USNM 1156944 & 1.11 & 0.87 & 0.27 \\
\hline USNM 1156944 & 1.11 & 0.87 & 0.29 \\
\hline USNM 1156944 & 0.96 & 0.78 & 0.27 \\
\hline USNM 1156944 & 1.07 & 0.80 & 0.24 \\
\hline USNM 1156944 & 1.11 & 0.89 & 0.27 \\
\hline MNHN 24596 & 1.07 & 0.87 & 0.22 \\
\hline MNHN 24596 & 1.07 & 0.87 & 0.22 \\
\hline MNHN 24596 & 1.09 & 0.80 & 0.22 \\
\hline
\end{tabular}

Paratypes. IBUFRJ 14991 (\# 54, 2002), 2 valves and 1 specimen; IBUFRJ 19176 (\# 64, 2003), 1 specimen; MZSP 99980 (\# 54, 2002), 5 valves; USNM 1156944 (\# 54, 2002), 5 valves; MNHN 24597 (\# 54, 2002), 2 valves 1 specimen.

Etymology. Nhanduti is a Tupi-Guarani term (the language spoken by the largest groups of native people living in Brazil prior to the European colonization) for a spider web-like structure, similar to those present on the prodissoconch of this species. The species epithet is proposed as a noun in apposition.

Material examined. IBUFRJ 15140 (\# 69, 2002), 17 valves and 2 specimens; IBUFRJ 15283 (\# 69, 2002), 6 valves; IBUFRJ 19115 (\# 33, 2002), 1 valve; IBUFRJ 19116 (\# 64, 2002), 16 valves and 1 specimen; IBUFRJ 19117, (\# 49, 2003), 7 valves; IBUFRJ 19118, (\# 54, 2003), 1 valve; IBUFRJ 19119, (\# 59, 2003), 10 valves and 2 specimens; IBUFRJ 19121, (\# 34, 2002), 1 valve; IBUFRJ 19122, (\# 37, 2002), 1 valve; IBUFRJ 19123, (\# 38, 2002), 1 valve; IBUFRJ 19124, (\# 64, 2003), 12 valves and 1 specimen; IBUFRJ 19125, (\# 75, 2003), 2 valves; IBUFRJ 19126, (\# A7, 2008), 4 specimens; IBUFRJ 19127, (\# A7, 2008), 1 valve; IBUFRJ 19128, (\# A7, 2008), 1 valve and 3 specimens; IBUFRJ 19129, (\# D7, 2008), 1 specimen; IBUFRJ 19130 (\# H7, 2008), 2 specimens; IBUFRJ 19131 (\# D6, 2008), 3 specimens; IBUFRJ 19132 (\# H7, 2008), 1 specimen; IBUFRJ 19133 (\# H7, 2008), 4 valves; IBUFRJ 19134 (\# D6, 2008), 1 specimen; IBUFRJ 19135 (\# I7, 2008), 1 specimen; IBUFRJ 19136 (\# A7, 2009), 2 specimens; IBUFRJ 19137 (\# A7, 2009), 6 specimens; IBUFRJ 19138 (\# D6, 2009), 4 specimens; IBUFRJ 19139 (\# H7, 2009), 10 specimens; IBUFRJ 19140 


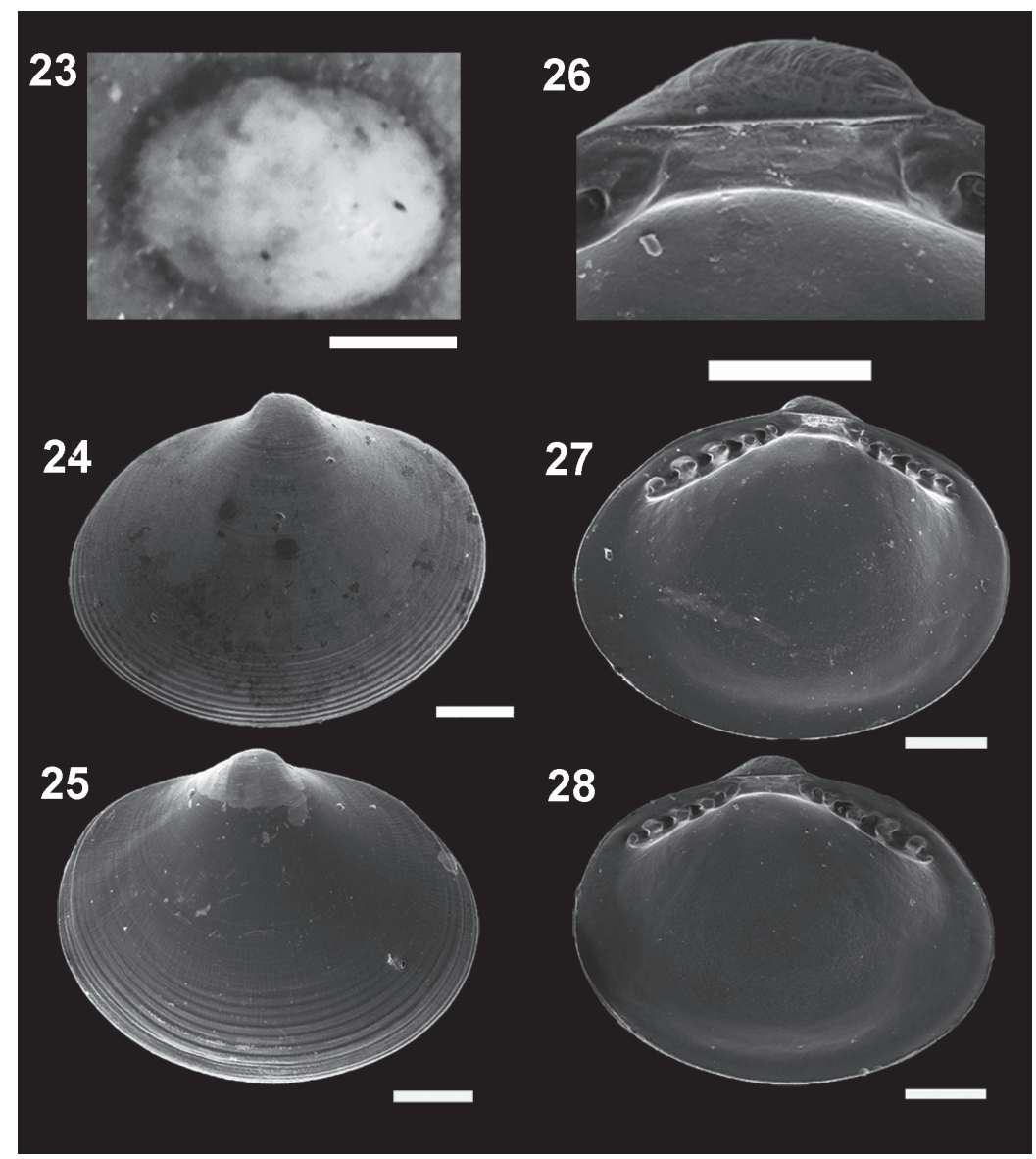

Figures 23-28. Microgloma pusilla (Jeffreys, 1879) USNM 19971223 Microgloma nhanduti sp. n. External view, leftt valve 24 IBUFRJ 15283, right valve 25 IBUFRJ 14991. Internal view holotype MNRJ 19.113, detail of the reslifer and umbo $\mathbf{2 6}$ right valve $\mathbf{2 7}$ left valve $\mathbf{2 8}$ Scale bars: $23=500 \mu \mathrm{m} ; 26=100$ $\mu \mathrm{m} ; 24-25,27-28=200 \mu \mathrm{m}$.

(\# CANAC7, 2009), 5 specimens and 2 valves; IBUFRJ 19141(\# CANAC7, 2008), 1 specimen; IBUFRJ 19142 (\# I7, 2008), 2 specimens; IBUFRJ 19143 (\# CANAC7, 2008), 6 specimens; IBUFRJ 19144 (\# D7, 2008), 1 specimen.

Diagnosis. Shell minute, ovate. Entire margin slightly thickened. Hinge plate moderately thick. Prodissoconch with web-like striae.

Description. Shell minute, ovate, H/L ratio about 0.78 (n 29, min 0.74, max 0.81 ), Width of both valves/ $\mathrm{H}$ ratio about 0.7 (n 11 , $\min 0.67$, max 0.77 ), inequilateral, translucent, glossy; umbones moderately prominent, large, posterior to midline, orthogyrous. Antero-dorsal margin convex and oblique; anterior margin rounded and projected. Antero-ventral margin convex, postero-ventral margin shorter and rising up to short and rounded posterior end; postero-dorsal margin convex, but forming a small shoulder. Entire shell margin slightly thickened. Surface with subtle commargin- 


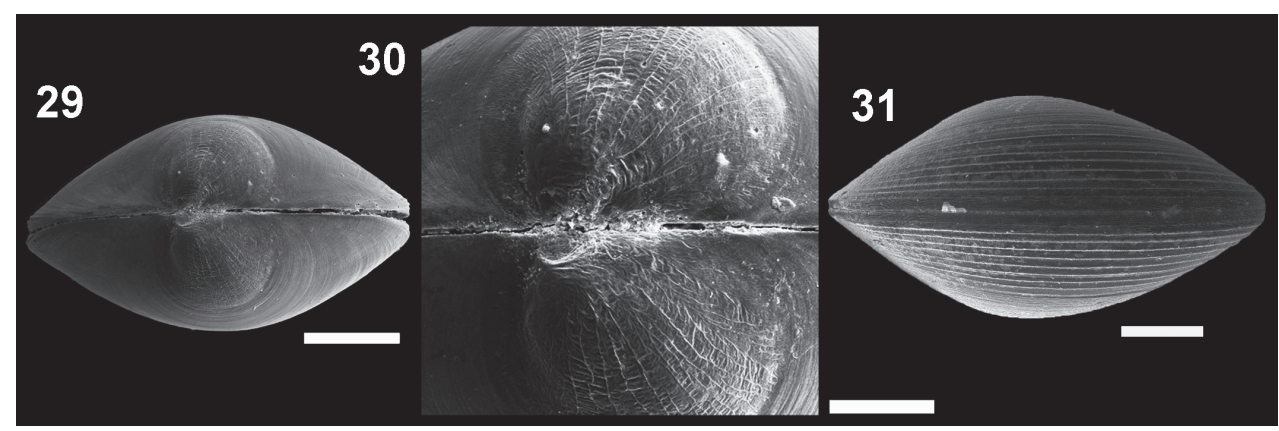

Figures 29-3I. Microgloma nhanduti sp. n. Dorsal view IBUFRJ 1913129 prodissoconch surface 30 ventral margin IBUFRJ 191763 I Scale bars: 29, $30=200 \mu \mathrm{m}, 31=80 \mu \mathrm{m}$.

al growth lines. Hinge plate with 5-7 anterior and 4-5 posterior teeth, interrupted by a shallow rectangular hinge plate. Width of both anterior and posterior row of teeth occupies about $70 \%$ of width of hinge plate, which is thick for its size (whp/ $\mathrm{H}$ approximately 0.14 ). Posterior part of the hinge plate shorter than anterior one. Prodissoconch surface nacreous, with several radial striae, resembling a spider's web. Prodissoconch length approximately $246 \mu \mathrm{m}$.Maximum adult shell length $1.16 \mathrm{~mm}$.

Remarks. Microgloma nhanduti sp. n. is similar to M. pusilla and M. tumidula in the oval outline. but has more prominent umbones (Figs 26-28). Compared to $M$. macaron sp. n. and M. yongei, $M$. nhanduti sp. n. has a more elongated outline, a longer anterior area, and a more evident prodissoconch sculpture, with a web-like pattern (Figs 29-30). In $M$. nhanduti sp. n. the umbo is not as prominent and the shell margin not as thick as in M. macaron sp. $n$. Untill now this species is reccorded solely in Campos Basin.

\section{Discussion}

Ockelmann and Warén (1998) carefully evaluated the systematic position of the Nuculoidea and Nuculanoidea, and placed Microgloma within the Nuculanidae (a position with which we agree). However, these authors did not use the subfamily rank introduced by Allen and Hannah (1986). Considering differences such as prominence of the rostral area, presence of carena and keels, foot grooves, and the characteristics of the ligament among some groups within the Nuculanidae such as in Ledella Verrill and Bush, 1897, Propeleda Iredale, 1924, and Nuculana Link, 1807, we believe that the proposed subfamilies should be used for taxonomic purposes. Whether they represent a natural division or not is a matter to be discussed later in a phylogenetic study.

The genus Microgloma is still in need of review since, as stated by Ockelmann and Warén (1998), "similarity in hinge structure to juvenile specimens of Yoldiella and other nuculanids directly suggests progenesis. We assume that the species of Microgloma simply are species derived from Yoldiella or Ledella, which mature at a much smaller size than normal in these taxa. (...) Possibly the genus Microgloma is polyphyletic, 
since progeneses may have taken place more than once. This will be difficult to prove or disprove." The similarities with some species of the genera cited above may confuse many researches in the identification of Microgloma species and the validity or status of the genus must be revisited. We believe this is an issue to be resolved with molecular analysis. At present we can only assume the genus to be valid.

The sculpture pattern on the prodissoconch surface is not a character commonly used in taxonomy of the protobranchs and, considering the confused taxonomy of the protobranchs (as seen by the genus Microgloma), we believe it might be useful to better determine the genera. This character has been recorded recently in the literature, and some species, from different families, show particular patterns. The reticulated sculpture on the prodissoconch surface seems to be a common character for the Nuculanidae, and has been recorded for the genera Nuculana, Sacella Woodring 1925 (Allen 1993, Ockelmann and Warén 1998, La Perna 2007), Propeleda (seen by Natalia Benaim; unpublished data), and the Bathyspinulidae, in Tindariopsis agathida Dall, 1889 (seen by the present authors; unpublished data). Some members of Nuculidae have ridges or knobs on the prodissoconch surface (Gofas and Salas 1996, Zardus 2002). The genus Yoldiella presents a smooth prodissoconch surface, but the species Yoldiella philippiana (Nyst, 1845) presents a radial prodissoconch sculpture with a web-like pattern (Ockelmann and Warén 1998, Salas 1996) as seen here in M. nhanduti sp. n. Salas (1996) also illustrated radial ridges on the prodissoconch of $M$. pusilla and $M$. tumidula from the Iberian Peninsula. The radial ridges present in $M$. macaron sp. n. and $M$. nhanduti sp. n. are distinctive characters that should be considered in future descriptions of species of Microgloma to aid in resolving the status of the genus.

\section{Conclusion}

The apparent absence of species of the genus Microgloma along the Brazilian coast wais an artifact, reflecting the logistical difficulties in obtaining material from these depths. Once this material became available, additions to the fauna was brought to light. The description of two new species of Microgloma and the new information on the conchology, and bathymetric and geographical distributions of $M$. mirmidina and $P$. alba contribute to knowledge of the biodiversity of deep-sea mollusks of the Campos Basin and Brazil.

\section{Acknowledgements}

We are indebted to Dr. John Allen for sending P. alba material, to Ellen Strong, Smithsonian Institution, for sending the image of Microgloma pusilla holotype, to Cleo Dilnei de Castro Oliveira for searching out material at the Museum of Comparative Zoology (Harvard University, USA), to the profitable suggestions made by the reviewers of this manuscript, and to Petrobras S.A for making this material available and for SEM support. 


\section{References}

Absalão RS (2009) New small deep-sea species of Gastropoda from the Campos Basin off Rio de Janeiro state, Brazil. American Malacological Bulletin 27: 133-140.

Absalão RS, Pimenta AD (2003) A new subgenus and three new species of Brazilian deep water Olivella Swainson, 1831 (Mollusca, Gastropoda, Olivellidae) collected by the RV Marion Dufresne in 1987. Zoosystema 25(2): 177-185.

Absalão RS, Pimenta AD (2005) New records and new species of Vetulonia Dall, 1913 and Brookula Iredale, 1912 from Brazil (Gastropoda, Trochidae). Veliger 47: 193-201.

Absalâo RS, Miyaji C, Pimenta AD (2001) The genus Brookula Iredale, 1912 (Gastropoda, Trochidae) from Brazil: description of a new species, with notes on other South American species. Zoosystema 23: 1-13.

Absalão RS, Caetano CHS, Pimenta AD (2003) Novas ocorrências de gastrópodes e bivalves marinhos no Brasil (Mollusca). Revista Brasileira Zoologia 20: 323-328. doi: 10.1590/ S0101-81752003000200024

Allen JA (2008) Bivalvia of the deep Atlantic. Malacologia 50(1-2): 57-173. doi: 10.4002/00762997-50.1.57

Allen JA (1993) A new deep-water hydrothermal species of Nuculana (Bivalvia: Protobranchia) from the Guaymas Basin. Malacologia 95: 141-151.

Allen JA, Hannah FJ (1986) A reclassification of the Recent genera of the subclass Protobranchia (Mollusca: Bivalvia). Journal of Conchology 32: 225-249.

Allen JA, Hannah FJ (1989) Studies on the deep sea Protobranchia. The subfamily Ledellinae (Nuculanidae). Bulletin of the British Museum of Natural History (Zoology) 55: 123-171.

Baylei JB (2009) Shell orientation terminology among the Bivalvia (Mollusca): problems and proposed solutions. Journal of Paleontology 83(3): 493-495. doi: 10.1666/08-111.1

Benaim NP, Absalão RS (2011) Deep sea Yoldiella (Pelecypoda, Protobranchia, Yoldiidae) from Campos Basin, Rio de Janeiro, Brazil. Journal of the Marine Biological Association of the United Kingdom 91(2): 513-529. doi: 10.1017/S0025315410001372

Benaim NP, Absalão RS (2011) Discriminating among similar deep-sea Yoldiella (Pelecypoda: Protobranchia) species with a morphometric approach. Journal of the Marine Biological Association of the United Kingdom 91: 1665-1672. doi: 10.1017/S0025315411000063

Benaim, NP, Viegas DCP, Absalão RS (2011) How features of the hinge plate aid in discriminating among three Yoldiella (Pelecypoda, Protobranchia) species from the Campos Basin, Brazil. Zootaxa 2883: 39-51.

Caetano CHS, Scarabino V, Absalão RS (2006) Scaphopoda (Mollusca) from the Brazilian continental shelf and upper slope $\left(13^{\circ}\right.$ to $\left.21^{\circ} \mathrm{S}\right)$ with description of two new species of the genus Cadulus Philippi, 1844. Zootaxa 1267: 1-47.

Clarke AH (1962) Annotated list and bibliography of the abyssal marine molluscs of the world. Bulletin of the National Museum of Canada 181: 1-114.

Dautzenberg P (1927) Mollusques provenant des campagnes scientifiques du Prince Albert ${ }^{\text {er }}$ de Monaco dans l'Océan Atlantique et dans le Golfe de Gascogne. Résultats des Campagnes Scientifiques accomplies sur son yacht par Albert $\mathrm{I}^{\text {er }}$ Prince Souverain de Monaco 72: 1-400. 
Dautzenberg P, Fisher H (1897) Campagnes Scientifiques de S.A.S. Le Prince Albert I ${ }^{\text {er }}$ de Monaco. Dragages effectués par l'HIRONDELLE et par La PRINCESSE ALICE. Mémoires de la Société Zoologique de France 9: 139-234.

Fischer PH (1886) Manuel de conchyliologie et de paléontologie conchyliologique. Histoire naturelle des mollusques vivants et fossiles, 10:897-1008.

Gofas S, Salas C (1996) Small Nuculidae (Bivalvia) with functional primary hinge in the adults. Journal of Conchology 35: 427-435.

La Perna R (2003) The Quaternary deep-sea protobranch fauna from the Mediterranean: composition, depth related distribution and changes. Bolletino Malacologico 39: 17-34.

La Perna R (2007) Revision on the Nuculanidae (Bivalvia: Protobranchia) from the Cerulli Irelli collection (Mediterranean, Pleistocene). Bolletino della società paleontologica Italiana 46(2-3): 125-137.

La Perna R (2008) Revision of the protobranch species described by Dautzenberg and Fischer (1897) with description of a new species and taxonomic comments on Bathyspinula (Bivalvia, Nuculanoidea). The Veliger 50 (2): 149-162.

Mikkelsen P, Bieler R (2007) Seashells of Southern Florida: Living Marine Mollusks of the Florida Keys and Adjacent Regions: Bivalves. University Press, Princeton, 496pp.

Ockelmann K, Warén A (1998) Taxonomy and biological notes on the Bivalve genus Microgloma with coments on protobranch nomenclature. Ophelia 48(1): 1-24.

Oliveira CDC, Absalão RS (2007) Primeiro registro de Mendicula ferruginosa, Kelliella atlantica e Lyonsiella subquadrata (Mollusca, Pelecypoda) para águas brasileiras. Biociências 15: 63-67.

Oliveira CDC, Absalão RS (2008) The genera Limatula and Limea (Mollusca, Pelecypoda, Limidae) from deep waters off Brazil. Zootaxa 1949: 48-58.

Oliveira CDC, Absalão RS (2009) The genera Myonera, Octoporea and Protocuspidaria (Pelecypoda, Cuspidariidae) from deep waters of Campos Basin, Rio de Janeiro, Brazil. With description of two new species. American Malacological Bulletin 27: 141-156. doi: 10.4003/006.027.0212

Oliveira CDC, Absalão RS (2010a) Review of the Septibranchia (Pelecypoda: Mollusca) from deep sea of Campos Basin, Brazil: family Verticordiidae, with description of a new species. Journal of the Marine Biological Association of the United Kingdom 90: 809-817. doi: $10.1017 /$ S0025315409991184

Oliveira CDC, Absalão RS (2010b) Review of the Septibranchia (Mollusca: Pelecypoda) from the deep sea of Campos Basin, Brazil: family Lyonsiellidae, with description of a new species. Scientia Marina 74: 305-316. doi: 10.3989/scimar.2010.74n2305

Oliver PG, Holmes AM, Killeen IJ, Turner JA (2009) Marine Bivalve Shells of the British Isles (Mollusca: Bivalvia). Amgueddfa Cymru - National Museum Wales. Available online at http://naturalhistory.museumwales.ac.uk/britishbivalves [accessed 03 May 2011]

Pimenta AD, Santos FN, Absalão RS (2008) Review of the genera Ividia, Folinella, Oscila, Pseudoscilla, Tryptichus and Peristichia (Gastropoda, Pyramidellidae) from Brazil, with description of four new species. The Veliger 50: 171-184.

Salas C (1996) Marine Bivalves from off the Southern Iberian Peninsula collected by the Balgim and Fauna 1 expeditions. Haliotis 25: 33-100. 
Sanders HL, Allen JA (1973) Studies on the deep sea Protobranchia (Bivalvia): Prologue and the Pristiglomidae. Bulletin of the Museum of Comparative Zoology 145(5): 237-261.

Zardus JD (2002) Protobranch Bivalves. Advances in Marine Biology 42: 1-65. doi: 10.1016/ S0065-2881(02)42012-3

Zelaya DG, Absalão RS, Pimenta AD (2006) A revision of Benthobrookula Clarke, 1961(Gastropoda, Trochoidea) in the south-western Atlantic Ocean. Journal of Molluscan Studies 72: 77-87. doi: 10.1093/mollus/eyi050 